\title{
Shimoji's Cranioplasty Yielding Life-quality Improvements in MIld Trigonocephaly (SCYLIMIT)
}

\begin{abstract}
In every textbook, it was written that mild trigonocephaly is not indicated for surgery due to its mild morphological changes of the skull and brain. We have had many patients of mild trigonocephaly with clinical symptoms and we have surgically treated them since 1994. The presentation will introduce our surgical results of the mild trigonocephaly.
\end{abstract}

Shimoji $T^{1}$

\section{1, Personal experiments}

The first several cases were treated for purely cosmetic reasons for moderate trigonocephaly. They had some milestone problems like language delays, hyperactivities and autistic tendencies. After surgery, patients' symptoms improved. At that time, it was felt that the research should be started to prove relationship between improvement of the patient's symptoms and surgical intervention.

The sixth case ( $3 \mathrm{y} / \mathrm{o}$, girl) diagnosed with mild trigonocephaly with intellectual disability and motor delays was evaluated by various examinations prospectively to improve her symptoms by surgery. After surgery, she made amazing improvement. This case strongly suggested that surgical treatment of mild trigonocephaly contributes to improvement of the patients' symptoms.

Since then, more than 541 cases have been treated. $97 \%$ of the patients have gotten some improvements in their symptoms. Especially, 74 patients among them got into normal class pertaining to their age of the primary school.

\section{2, Mechanism}

Features of the cases treated by us:

Pre-operatory 3D-CTs show metopic ridge, smaller frontal area and anterior fossa with marked digital markings.

Pre-operatory MRIs show no abnormal findings in the brain except e smaller frontal lobes.

During surgery, the abnormal overgrowth of the sphenoid ridges have been found. Large part of the sphenoid bones and frontal calvarial bones were removed. After making one piece of the orbital bar, it is advanced foreword to several centimeters.

This method gives to the patients an enlarged frontal area and anterior fossa resulting in the frontal lobes growing bigger especially pars opercularis. Also, the digital markings disappear due to decrease in the intracranial pressure.

It is thought that deleting the elements of dis-functioning of the brain improves their symptoms.

\section{$\underline{\text { 3, Quantification }}$}

Several psychological tests were applied to find whether the surgical intervention makes patients' status better or not. The prospective studies accomplished showed that every test had statistically positive results.

\section{Sponsors}

The Just Beyond Medicine (JBM) Oration-2018 was sponsored by JBM Inc., Japan

The lecture was delivered in the Just Beyond Medicine (JBM) Oration-2018 session of NCRM NICHE (www.ncrmniche.org) which is a day of commemorative events to celebrate the inaugural Anniversary of Nichi-In Centre for Regenerative Medicine, conducted every year since 2006 in the month of October. NCRM NICHE provides a platform that augments the interaction of scientists and clinicians. It enables them to exchange ideas in order to arrive at synergies while working towards a common goal of discovering clinically applicable solutions for diseases without a definitive treatment to yield relief to numerous patients. Conducted in India till 2016, it has been conducted in Japan from 2017 onwards. NCRM NICHE has two components: 1. Active Knowledge Gaining (AKG) events which refer to any knowledge gaining activity in which the participant plays an active role before and during the event instead of being a passive listener to a lecture by a speaker or a conversation among third parties. AKG events of NCRM NICHE include a. Fujio Cup Quiz (FCQ); b. Oral Presentation session and c. Tour to Premier Institutes and Inter Disciplinary Conclave (IDC); 2. Passive Knowledge Gaining $(P K G)$ events which refer to an event, where there is no mandatory preparation by the participants before the event. During the event, they have to merely be a passive listener to others either delivering a lecture or interacting among third parties. PKG events of NCRM NICHE include a. Lectures \& Orations and b. Inter-Disciplinary Interactions and Solutions (IIDIAS) Session. NCRM NICHE 2018 was conducted at the Shibaura Institute of Technology, Tokyo, Japan from $21 \sim 23$ October 2018. 